\title{
Factores predictores del modelo de negocio validado por directivos de pequeñas y medianas empresas de Monterrey, Nuevo León
}

\section{Predictive factors of the business model validated by managers of small and medium-sized companies in Monterrey, Nuevo León}

\author{
José Antonio Sánchez Valdez ${ }^{1}$, Omar Arodi Flores Laguna², \\ Karla Saraí Basurto Gutiérrez*1,2 \\ ${ }^{1}$ Tecnológico Nacional de México, México \\ ${ }^{2}$ Universidad de Montemorelos, México
}

Recibido el 15 de noviembre de 2018; aceptado el 9 de marzo de 2020

Disponible en Internet el: 13 de marzo de 2020

\section{Resumen}

Esta investigación estudió las relaciones de colaboración, comportamiento estacional de la de-manda y capital intelectual y, cómo estas variables predicen la práctica de un modelo de nego-cio, según la percepción de directivos de Pymes de Monterrey, Nuevo León, México.

Este estudio es de tipo cuantitativo y predictivo. La muestra que se utilizó fue de 233 directivos de Pymes de Monterrey, Nuevo León, México. Se realizó un análisis de regresión lineal por el método de pasos sucesivos, mediante el cual se encontró que las variables capital intelectual y comportamiento estacional de la demanda explican en un $76 \%$ la varianza de la variable criterio modelo de negocio.

Código JEL: M10, M13, M19

Palabras clave: Relaciones de colaboración; Comportamiento estacional de la demanda; Capital intelectual; Modelo de negocio; Innovación empresarial

\footnotetext{
*Autor para correspondencia. Correo electrónico: kbasurto@um.edu.mx (K. Basurto Gutiérrez).

La revisión por pares es responsabilidad de la Universidad Nacional Autónoma de México.
} 


\begin{abstract}
This research studies collaborative relationships, the seasonal behavior of demand and the in-tellectual capital and how these variables predict the business model, according to the percep-tion of the leaders of SMEs in Monterrey, Nuevo León, Mexico.

This study is quantitative and predictive. A sample of 233 SME managers from Monterrey, Nuevo León, Mexico was used. The $74.7 \%$ of the variance dependent business model was carried out, a linear regression analysis by the method of stepwise. It has also been found that the variables of intellectual capital and seasonal behavior of demand are good predictors ex-plained by $76 \%$ variance of the variable criterion business model.
\end{abstract}

JEL Code: M10, M13, M19

Keywords: Collaborative relationships; Seasonal behaviour of demand; Intellectual capital; Business model; Business Innovation

\title{
Introduction
}

Las relaciones de colaboración, el comportamiento estacional de la demanda, el capital intelectual y la práctica del modelo de negocio son factores clave para el éxito en la empresa que no se estudian en conjunto; la mayoría de los autores los han estudiado de forma separada y reconocen que son importantes en la empresa (Spreitzer, 1995; Giraldo Marín, 2012; Bozart, Warsing, Flynn y Flynn, 2009 y Villena Manzanares y Souto Pérez, 2015). Por otro lado, el Instituto Nacional de Estadística y Geografía (INEGI, 2016) señala como una necesidad que las empresas tengan inversiones potenciales en innovación y que estas se conviertan en oportunidades de negocio, mejorando los procesos para lograr relaciones a largo plazo con sus clientes. Cabe destacar que las Pymes constituyen la columna vertebral de la economía nacional por los acuerdos comerciales que ha tenido México en los últimos años y, asimismo, por su alto impacto en la generación de empleos y en la producción nacional. En México existen aproximadamente 4 millones 15 mil unidades empresariales, de las cuales, el 99.8\% son Pymes que generan el 52\% del producto interno bruto y el $72 \%$ del empleo en el país. Tal es el ejemplo de Monterrey, una de las ciudades más desarrolladas de México y principal centro de negocios del país. Es la ciudad con mejor calidad de vida en México, la novena en América Latina y la $112^{\mathrm{a}}$ en el mundo. En este lugar radican muchas empresas nacionales e internacionales como Cemex, Oxxo, FEMSA, Vitro, Grupo Alfa, entre otras, por lo que es llamada La Capital Industrial de México. 
Asimismo, autores como Camisón Zornoza, Garrigós Simón y Palacios Marqués (2007) y Garcés, López y Pailiacho (2017) enfatizan que los modelos de negocio reducen la compleja estructura de las organizaciones resumiéndola en categorías y que, estas, son una guía para los directivos, ya que los ayuda a entender de forma resumida cómo una compañía gana dinero, genera valor y ofrece un servicio o producto con valor. También, Macri, Tagliaventi y Bertolotti (2002) afirman que una empresa es más emprendedora cuando consigue identificar y explotar nuevas oportunidades en el modelo de negocio. Además, Den Hertog, Van der Aa y de Jong (2010), agregan que, con el modelo de negocio definido, se pueden plantear nuevas formas de valorar las capacidades de innovación con un marco conceptual y teórico para la gestión y administración; es por ello que el propósito de este estudio es evaluar la percepción de los directivos de algunas Pymes de Monterrey, Nuevo León, México en sus relaciones de colaboración, comportamiento estacional de la demanda y capital intelectual como predictores del modelo de negocio . La investigación del conocimiento sobre los motivos que originan las diferencias de resultados entre organizaciones sigue siendo uno de los temas centrales y más desafiantes del estudio (Claver Cortés, Pertusa Ortega y Molina Azorín, 2010).

\section{Revisión literaria}

La primera variable en estudio fue relaciones de colaboración; al respecto, Faust, Christens, Sparks y Hilgendorf (2015) señalan que las nuevas modalidades de colaboración involucran flujos de recursos bilaterales, asî́ como esfuerzos de planeación y estrategia de ambos sectores. Lee y Choi (2003) mencionan que las empresas que operan en sectores industriales dinámicos y de alta rivalidad enfrentan fuertes presiones para ofrecer en los mercados productos atractivos por su precio y calidad. Para lograrlo, desde el punto de vista de Giraldo Marín (2012) las empresas deben permitir y apoyar el empoderamiento de sus empleados, ya que cuando se empodera a un empleado es porque se piensa en él como una persona con alto potencial creativo, conocimiento e iniciativa para desarrollar y contribuir a los fines organizacionales. Por otro lado, Martín Ríos y Septiem (2013) señalan que, para que las empresas puedan tener éxito en el contexto económico abierto y competitivo actual, no es suficiente canalizar y administrar recursos internos con los que desarrollar productos y servicios. Con frecuencia las empresas se enfrentan a la necesidad de colaborar con otras empresas, incluso competidoras, para obtener información sobre aspectos esenciales como las formas de organizar el trabajo, de innovar y de producir u ofrecer servicios. Para esta investigación relaciones de colaboración se define como el proceso donde se involucra el trabajo de varias personas en conjunto, tanto para conseguir un resultado muy difícil de realizar individualmente como para ayudar a conseguir algo a quien por sí mismo no podría. 
La segunda variable en estudio fue comportamiento estacional de la demanda; para Alonso y Arcila (2013), es la documentación de la estacionalidad en el comportamiento de los precios en mercados, tanto de activos financieros como de materias primas; conocer esta información genera una ventaja competitiva ya que los aspectos por evaluar deberán ser estacionalidad, tendencia, variación aleatoria y variación cíclica, haciendo necesario realizar un análisis del mercado en segmentos y tratar de pronosticar un periodo tomando en cuenta todos los factores para que esta sea satisfactoria. Del mismo modo, Bozart, Warsing, Flynn y Flynn (2009) y Lavanda y Rodríguez (2011) mencionan que los modelos de demanda y oferta agregada determinan el nivel de precios y la producción, dando un conjunto de variables. Asimismo, dichos modelos permiten la realización de distintos análisis temporales por medio de sus respectivas variantes para un análisis de corto, mediano y largo plazo. Un aspecto importante para resaltar en esta variable de acuerdo con Cuevas Vargas, Aguilera Enríquez, González Adame y Servín (2015) es que las Pymes, enfrentan múltiples desafíos para poder permanecer en los mercados globales altamente competitivos y cambiantes por los efectos del comportamiento de la demanda que actualmente enfrentan. Para este trabajo de investigación el comportamiento estacional de la demanda se define como la estadística de un determinado producto que muestra estacionalidad cuando la serie de tiempo subyacente atraviesa una variación cíclica predecible según la época del año

La tercera variable en estudio es el capital intelectual. El capital intelectual comprende todos los conocimientos tácitos y explícitos que generan valor económico para la entidad (Brooking, 1997; Sagástegui, 2014). Para Secundo, Dumay, Schiuma y Passiante (2016), el CI es un concepto multi-dimensional de los activos de conocimiento, experiencia y capacidades prácticas para crear valor.

Varios autores dividen al capital intelectual de la siguiente manera: (a) capital humano, (b) estructural y (c) relacional (Bontis, Chua y Richardson, 2000; Wee y Chua, 2016). Reyes (2011) menciona que el capital humano es el talento que tienen las personas y es el principal valor que tienen las organizaciones. El capital estructural comprende los activos estratégicos más valiosos de la empresa, como capacidades organizacionales, culturales, procesos, patentes, derechos de autor, marcas registradas y bases de datos (Denicolai, Ramusino y Sotti, 2015; Hejazi, Ghanbari y Ali-pour, 2016). Por último, Alvarez y Gonzáles (2013) dividen el capital relacional en agentes externos e internos. Los agentes externos son los clientes, proveedores, vendedores y administraciones públicas. Los agentes internos tienen que ver con las buenas relaciones con los accionistas, directivos, trabajadores, mercado (imagen y logotipo), reputación, ética y marca. En resumen, el capital intelectual se define como el grado de competencia requerido en las funciones de una ocupación, obtenido como combinación de factores que determinan la complejidad, el grado de autonomía y responsabilidad y las exigencias de conocimiento propios del desempeño idóneo de esas funciones. 
La cuarta y última variable en estudio para esta investigación es el modelo de negocio. Según Manrique Henao, Robledo Velásquez y Lema Tapias (2014), en las últimas décadas, la posibilidad de acceder a grandes cantidades de información en los países desarrollados ha permitido la formulación de metodologías y modelos de análisis de la innovación para las empresas. Por consiguiente, Garcés, López y Pailiacho (2017) definen que un modelo de negocio es una herramienta conceptual que, mediante un conjunto de elementos y sus relaciones, permite expresar la lógica mediante la cual una compañía intenta ganar dinero generando y ofreciendo valor a uno o varios segmentos de clientes, mediante la arquitectura de la firma, su red de aliados para crear, mercadear y entregar este valor, y el capital relacional para generar fuentes de ingresos rentables y sostenibles. El ciclo gerencial en el que se construye un plan comercial generalmente da por hecho el modelo de negocio y se ocupa en formular estrategias que logren una posición competitiva ventajosa dentro de los límites del modelo; esta práctica se puede enriquecer si se combina con el diseño de modelos de negocio. Para esta investigación modelo de negocio se refiere a la representación abstracta de una organización, ya sea de manera textual o gráfica, de todos los conceptos relacionados, acuerdos financieros, así como el portafolio central de productos o servicios que la organización ofrece y ofrecerá con base en las acciones necesarias para alcanzar las metas y objetivos estratégicos

\section{Sustento teórico del modelo}

De Oliveira Cabral, Fernandes Mateos Coelho, Fernandes Coelho y Braga Costa (2015) presentan un modelo que fue probado con datos de 498 empresas exportadoras distribuidas en todos los sectores manufactureros brasileños, por tamaño de empresa. La investigación muestra una relación fuerte entre las relaciones de colaboración, capital intelectual y el modelo de negocio, pero no impacta en la innovación del producto. Los datos fueron recogidos a través de un cuestionario. Este cuestionario respondió a 38 preguntas, en una escala Likert de siete puntos, con 1 significando "no en absoluto" y 7 "en extremo". Como resultado, se encontró evidencia de que existe una relación fuerte entre las relaciones de colaboración, el capital intelectual y el modelo de negocio.

En la investigación empírica de Villena Manzanares y Souto Pérez (2015), se analizó las Pymes manufactureras. La muestra incluía 150 Pymes de la provincia de Sevilla (España). El modelo de investigación incorpora las siguientes variables: capital relacional, cultura sostenible, certificación de calidad, capacidad de mejora competitiva, capacidad organizativa y orientación hacia la I+D (importancia que tiene para la empresa el disponer de tecnologías que mejore las características de sus productos o procesos productivos), formando parte del nivel de capital intelectual. El análisis realizado permitió concluir que, tanto el capital relacional orientado al comercio exterior como la cultura sostenible de la empresa, la certificación 
de calidad, la capacidad de mejora competitiva y la orientación hacia la $\mathrm{I}+\mathrm{D}$, impactan de manera positiva en el desempeño exportador de la empresa (modelo de negocio). También concluyeron que otras características internas de las empresas analizadas, como son la cultura sostenible y la capacidad de mejora competitiva, inciden sobre el desempeño exportador de una manera muy similar.

García Osorio, Quintero Quintero y Arias Pérez (2014) realizaron una investigación cuantitativa de corte transversal, empírico y explicativo, utilizando un instrumento para la recolección de la información empírica enviado a una base de datos de empresas de servicios en Colombia a los correos electrónicos de sus directivos, el tamaño de la muestra fue de 384, donde, finalmente, se obtuvieron 335 respuestas, de las cuales 318 son válidas. Esta investigación se enfocó en el sector servicios, debido a su importancia y dinamismo dentro de la economía colombiana. La recolección de información permitió establecer los valores de los constructos capacidad de la innovación de orientación al cliente (CIOC), capacidad de innovación de orientación la mercadeo (CIOM), capacidad de innovación de orientación a la tecnología (CIOT), desempeño innovador (DI), desempeño financiero (DF) y desempeño no financiero $(\mathrm{DnF})$, con el propósito de establecer relaciones entre ellos. El principal hallazgo que arroja el análisis consiste en demostrar que las capacidades de innovación (CI) relacionadas con la tecnología no tienen incidencia sobre el desempeño innovador en empresas de servicios. Para fines de esta investigación, el capital intelectual influye en el modelo de negocio y este, a su vez, en el desempeño empresarial.

Sin duda alguna, el desequilibrio en la demanda, las relaciones de colaboración y el capital intelectual son variables indispensables y estudiadas por varios autores. Como se mencionó anteriormente el propósito de esta investigación fue analizar los modelos de negocios de Pymes en Monterrey, Nuevo León, México, donde el problema de investigación consiste en conocer si las relaciones de colaboración (RC), el comportamiento estacional de la demanda (CED) y el capital intelectual (CI) son predictores significativos del modelo de negocio (MN), según la percepción de directivos en Pymes de Monterrey, Nuevo León, México (ver Figura 1).

\section{Metodología}

Este estudio tiene un enfoque cuantitativo y predictivo. La población utilizada en esta investigación estuvo formada por 2500 Pymes registradas en CAINTRA en el estado de Nuevo León, México entre los meses de septiembre a diciembre del año 2017. El tipo de muestreo realizado en esta investigación fue no aleatorio y por conveniencia, ya que se seleccionó a los directivos de las empresas de Monterrey, Nuevo León, México y su área metropolitana que estuvieron dispuestos a participar. La muestra fue de 233 directivos, que representan el 
9.32\% de la población y tiene las siguientes características: (a) en relación con la edad, de 20 a 30 años, el $48.7 \%$; de 31 a 40, el 29.3\%; de 41 a 50, el $18.5 \%$ y de 51 a 60 , el $3.4 \%$; (b) con respecto al sexo, el $79.3 \%$ son hombres y el $20.7 \%$, mujeres; (c) sobre el nivel académico, el $14.2 \%$ estudiaron hasta preparatoria; el $69.4 \%$, hasta licenciatura y hasta el posgrado, solo el 16.4\%; (d) en relación a su área de desempeño, el 3.9\% lo hace en ventas; el 34.3\%, en producción; el $8.2 \%$, en compras; el 12.4\%, en administración y, en otras áreas, el 41.2\%; (e) con respecto al puesto que tienen, el 17\% trabaja en el nivel operativo; el 59.2\% es supervisor; el $22.9 \%$ de ellos trabajan en la gerencia y, en la dirección, solo el .9\%; (f) en relación al sector, el $4.8 \%$ está en ventas; en fabricación, el $88.3 \%$ y, en servicios, el 6.9\%. Para la recolección de los datos se usó un cuestionario creado por Sánchez Valdez (2018) con una escala tipo Likert en donde 1 es nunca, 2 es casi nunca, 3 es a veces, 4 es casi siempre y 5 es siempre. Cada constructo del cuestionario utilizado está dividido en tres dimensiones y lo conforman 16 declaraciones.

La confiabilidad del instrumento se midió por variable con su correspondiente alfa de Cronbach: (a) relaciones de colaboración, .936, (b) comportamiento estacional de la demanda, .930 , (c) capital intelectual, .934 y (d) modelo de negocio, .934 .

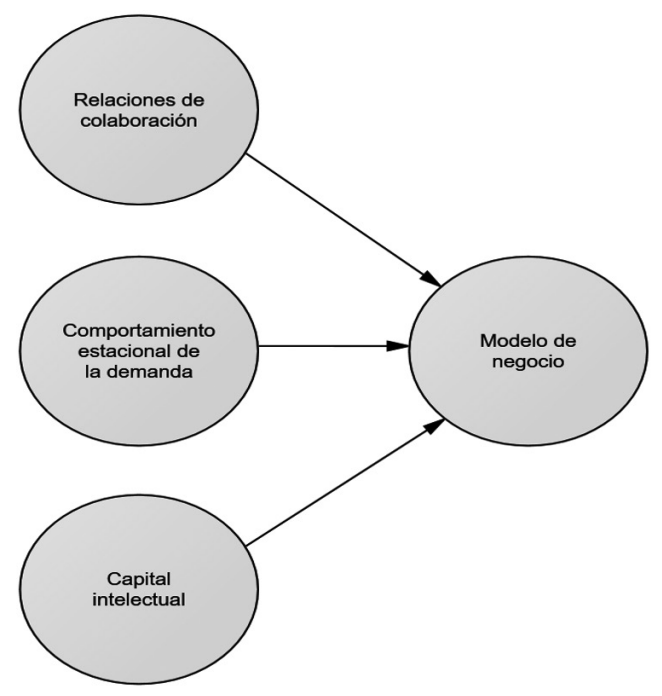

Figura 1. Modelo de investigación. Fuente: Elaboración propia. 


\section{Resultados}

En esta sección, se presentan los resultados de las medias aritméticas y desviación estándar de cada constructo y sus ítems. Mediante el análisis de las respuestas provistas por 233 directivos, se obtuvo una media aritmética para relaciones de colaboraciones de $(M=4.28, S$ $=.743)$, comportamiento estacional de la demanda $(M=4.07, S=.742)$, capital intelectual $(M=3.92, S=.756)$ y modelo de negocio $(M=3.60, S=.807)$.

Para profundizar en el estudio sobre cómo los directivos trabajan de manera práctica las variables en estudio, se obtuvieron las medias aritméticas de las dimensiones (ver Tabla 1), en donde se puede observar que la dimensión más baja en relaciones de colaboración es trabajo en equipo (RCB) y, la más alta, información estratégica (RCA). Para la variable competencia estacional de la demanda la dimensión más baja es transmisión de conocimiento (CEDA), la más alta fue capacidad tecnológica (CEDC). Para capital intelectual la dimensión más baja es capital relacionado a la organización (CIC) y, la más alta, capital humano (CIA). Para modelo de negocio la más baja es la organización que investiga, innova e involucra. En el anexo se encuentran todas las medias aritméticas de las declaraciones.

\section{Supuestos de regresión múltiple}

Hair, Anderson, Tatham y Black (2007) mencionan cuatro supuestos a probar en la regresión múltiple que son las siguientes: (a) linealidad del fenómeno, (b) normalidad de los residuos, (c) independencia de los términos de error y (d) varianza constante del término de error (homocedasticidad). Para esta investigación, el primer criterio que se analizó fue el de linealidad de cada variable independiente con la variable criterio y se observó en los gráficos de dispersión que existe una relación positiva y los puntos tienden a ser una línea recta (ver Figura 2). El segundo criterio que se probó fue la normalidad de los errores utilizando el estadístico de Kolmogorov-Smirnov $(p>.05)$; se eliminaron siete datos atípicos y se probó que la distribución de los residuos es normal $(p=.200)$ (ver Tabla 2). En el tercer criterio se probó la independencia de los errores, utilizando la prueba de Durbin-Watson cuyo valor fue de $D W$ $=1.970$ el cual está muy próximo a dos; esto indica que los errores no están correlacionados y son independientes (ver Tabla 3). Por último, se analizó la homocedasticidad, utilizando el gráfico del valor predicho estandarizado y el valor del residuo estandarizado; se observó que no hay relación lineal en los residuos y, por lo tanto, los errores tienen varianzas iguales (ver Figura 3). 
Tabla 1

Medias aritméticas por dimensiones

\begin{tabular}{lcc}
\hline & M & S \\
\hline RCA (Información estratégica) & 4.24 & .754 \\
RCB (Trabajo en equipo) & 4.16 & .763 \\
RCC (Compromiso laboral) & 4.17 & .689 \\
RCtotal & 4.19 & .646 \\
CEDA (Transmisión de conocimiento) & 3.80 & .819 \\
CEDB (Capacidad reactiva) & 4.05 & .736 \\
CEDC (Capacidad tecnológica) & 4.24 & .681 \\
CEDtotal & 4.04 & .658 \\
CIA (Capital humano) & 3.94 & .718 \\
CIB (Capital estructural) & 3.93 & .812 \\
CIC (Capital relacionado a la organización) & 3.82 & .825 \\
CItotal & 3.90 & .704 \\
MNA (Creatividad del capital humano) & 3.59 & .752 \\
MNB (La organización que investiga, innova e involucra) & 3.46 & .879 \\
MNC (El desempeño innovador) & 3.56 & .928 \\
MNtotal & 3.54 & .750 \\
\hline
\end{tabular}

Fuente: Elaboración propia.

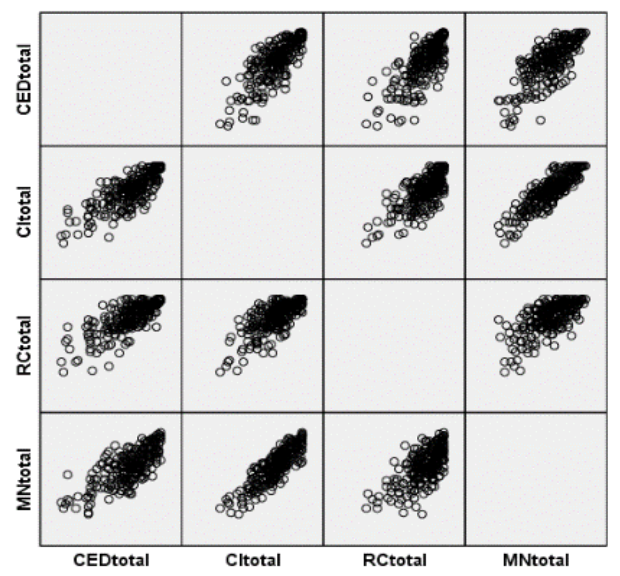

Figura 2. Linealidad con la variable criterio.

Fuente: Elaboración propia. 
Tabla 2

Prueba de normalidad

\begin{tabular}{lccccccc}
\hline & \multicolumn{3}{c}{ Kolmogorov-Smirnov } & \multicolumn{3}{c}{ Shapiro-Wilk } \\
& Estadístico & Gl & Sig. & Estadístico & Gl & Sig. \\
\hline Standardized Residual & .039 & 226 & $.200^{*}$ & .989 & 226 & .073 \\
\hline
\end{tabular}

Fuente: Elaboración propia

Tabla 3

Prueba Durbin- Watson

\begin{tabular}{lccccc}
\hline Modelo & $\mathrm{R}$ & R cuadrado & $\begin{array}{c}\text { R cuadrado } \\
\text { corregida }\end{array}$ & $\begin{array}{c}\text { Error típ. de la } \\
\text { estimación }\end{array}$ & Durbin-Watson \\
\hline 1 & $.865^{\mathrm{a}}$ & .748 & .747 & .37762 & \\
2 & $.872^{\mathrm{b}}$ & .761 & .759 & .36888 & 1.970 \\
\hline
\end{tabular}

Fuente: Elaboración propia

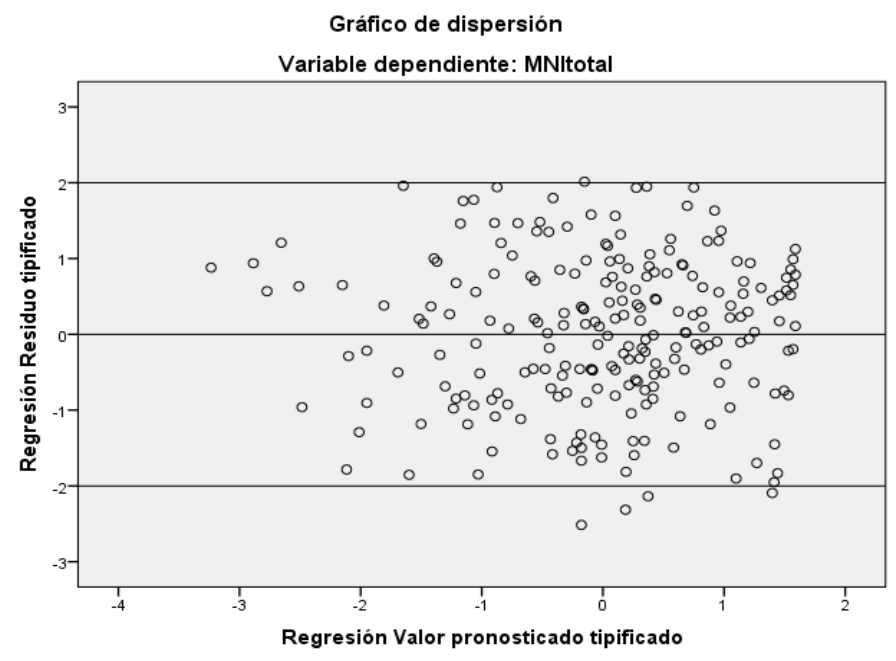

Figura 3. Gráfico de dispersión.

Fuente: Elaboración propia 


\section{Prueba de hipótesis nula}

$\mathrm{H}_{0}$ : El grado de relaciones de colaboración, el comportamiento estacional de la demanda y el capital intelectual no son predictores del modelo de negocio percibido por directivos de empresas pequeñas y medianas en Monterrey, Nuevo León.

Para el análisis de esta hipótesis, se utilizó la técnica estadística de regresión lineal múltiple por el método de pasos sucesivos; se consideraron como variables independientes el grado de relaciones de colaboración, el comportamiento estacional de la demanda y el capital intelectual y, como variable dependiente, el modelo de negocio.

El modelo se expresa de la siguiente manera:

$$
\begin{gathered}
\mathrm{y}=\beta_{0}+\beta_{1} x_{1}+\beta_{2} x_{2}+\beta_{3} x_{3}+\varepsilon_{1} \\
\mathrm{MN}=\beta_{0}+\beta_{1}(\mathrm{RC})+\beta_{2}(\mathrm{CED})+\beta_{3}(\mathrm{CI})
\end{gathered}
$$

Donde:

$\mathrm{y}=$ modelo de negocio

$\mathrm{x}_{1}=$ relaciones de colaboración

$\mathrm{x}_{2}=$ comportamiento estacional de la demanda

$\mathrm{x}_{3}=$ capital intelectual

Al realizar el análisis de regresión por el método de pasos sucesivos, se encontró que el mejor predictor fue la variable capital intelectual explicando el $74.7 \%$ de la varianza de la variable dependiente modelo de negocio (ver el modelo 1, en la Tabla 4). También se observó que las variables capital intelectual y comportamiento estacional de la demanda son buenos predictores del modelo de negocio (la variable relaciones de colaboración no fue significativa). El valor de $R^{2}$ corregida fue igual a .759 , lo cual indica que estas dos variables explican en un $76 \%$ la varianza de la variable dependiente modelo de negocio (ver el modelo 2, en la Tabla 4).

De igual manera, en el modelo 2 propuesto que se muestra en la Tabla 4 se obtuvo el valor $F$ igual a 354.726 y el valor $p$ igual a .000 . Como el nivel de significación es menor a .05 , se indica que existe una correlación lineal positiva y significativa. Con base en lo anterior, se rechaza la hipótesis nula. 
Tabla 4

Resultados del análisis de regresión

\begin{tabular}{clcccc}
\hline Modelo & & Regresores & $\mathrm{R}$ & $\mathrm{R}^{2}$ & Ajuste de $\mathrm{R}^{2}$ \\
\hline 1 & CI & .865 & .748 & .747 \\
2 & CI y CED & .872 & .761 & .759 \\
\hline
\end{tabular}

Fuente: Elaboración propia.

Los valores del coeficiente no estandarizado $B k$, obtenidos mediante la técnica estadística de regresión, fueron los siguientes: $B_{0}$ igual a - $.300, B_{1}$ no significativo, $B_{2}$ igual 205 y $B_{3}$ igual a .772. Con dichos valores se pudo construir la siguiente ecuación de regresión, utilizando el método de mínimos cuadrados:

$$
\mathrm{MN}=-.300+.205(\mathrm{CED})+.772(\mathrm{CI})
$$

En la figura 4 se muestran los coeficientes estandarizados para comportamiento estacional de la demanda $B$ igual a .179 y el capital intelectual $B$ igual .725. También se analizó la colinealidad de los constructos y se observó que el factor de la inflación de la varianza de comportamiento estacional de la demanda y capital intelectual fue menor que diez (FIV = 2.558), por lo cual, se concluye que los constructos modelo de negocio y comportamiento estacional de la demanda no presentan colinealidad.

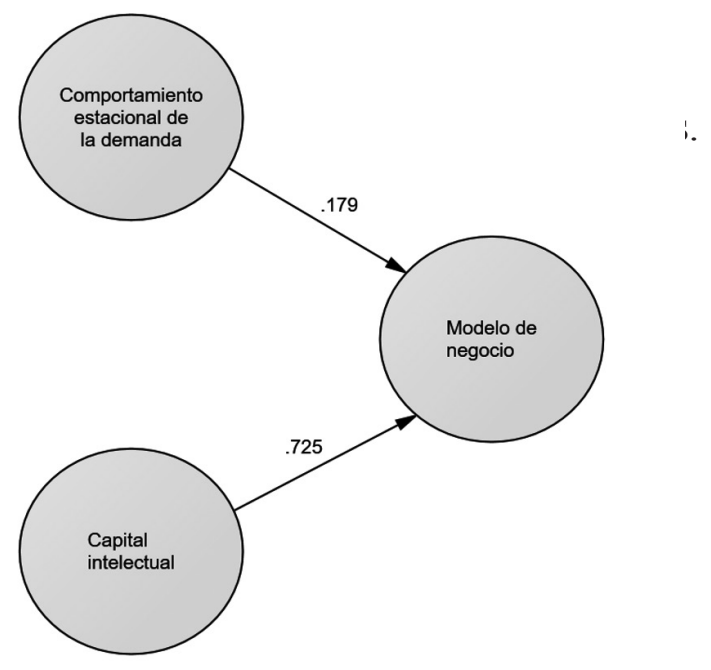

Figura 4. Modelo final con resultados.

Fuente: Elaboración propia 
En la Tabla 5 se analizaron las dimensiones de cada constructo que mejor explican el modelo de negocio encontrándose que CIC (Capital relacionado a la organización), CIA (Capital humano), CIB (Capital estructural) y CEDA (Transmisión de conocimiento) son las dimensiones que explican significativamente a la variable modelo de negocio.

Tabla 5

Coeficientes de los modelos

\begin{tabular}{lccccc} 
& \multicolumn{2}{c}{ Coeficientes no estandarizados } & Coeficientes tipificados & \\
\cline { 2 - 4 } Modelo & $\mathrm{B}$ & Error típ. & Beta & T & Sig. \\
\hline (Constante) & -.219 & .182 & & -1.199 & .232 \\
RCA & .088 & .047 & .088 & 1.858 & .065 \\
RCB & .002 & .052 & .002 & .036 & .971 \\
RCC & -.110 & .058 & -.101 & -1.884 & .061 \\
CEDA & .119 & .052 & .129 & 2.294 & $.023^{*}$ \\
CEDB & .070 & .053 & .068 & 1.317 & .189 \\
CEDC & .007 & .058 & .007 & .127 & .899 \\
CIA & .311 & .059 & .298 & 5.247 & $.000^{* * *}$ \\
CIB & .172 & .057 & .186 & 3.019 & $.003^{* * *}$ \\
CIC & .307 & .048 & .337 & 6.427 & $.000^{* * *}$ \\
\hline
\end{tabular}

Variable dependiente: MNtotal, $(* \mathrm{p}<.05, * * \mathrm{p}<.01, * * * \mathrm{p}<.001)$

Fuente: Elaboración propia

\section{Conclusión}

El Banco de México (2006) menciona que las actividades de investigación, desarrollo e innovación deben reforzarse para mejorar la competitividad. Con respecto a esta necesidad esta investigación pretendió dar respuesta a la siguiente pregunta: los constructos relaciones de colaboración, comportamiento estacional de la demanda y capital intelectual ¿son predictores significativos del modelo de nego- 
cio, según la percepción de directivos de Pymes en Monterrey, Nuevo León, México? En relación a la pregunta de investigación, los resultados muestran que el capital intelectual por si solo explica el $75 \%$ del modelo de negocio y con la variable comportamiento estacional de la demanda explican el $76 \%$.

Estos resultados concuerdan con los obtenidos por algunos autores que han realizado estudios similares. Cassol, Goncalo, Santos y Ruas (2016) realizaron una investigación, con el objetivo de analizar un modelo de gestión estratégica del capital intelectual en las prácticas de la capacidad de absorción como potenciador de la innovación de los modelos de negocio. Los resultados mostraron que la percepción de los gestores del capital intelectual se convierte en un motor de la innovación. Por lo tanto, esta investigación aporta como contribución la evidencia empírica de que el capital intelectual puede ser promovido por prácticas en la capacidad de potenciar la innovación, demostrando la existencia de la relación entre capital intelectual y modelos de negocio. Igualmente, De Oliveira Cabral, Fernandes Matos Coelho, Fernandes Coelho y Braga Costa (2015) encontraron una relación fuerte entre el capital intelectual y el modelo de negocio.

García Cruz y Real Fernández (2013) estudiaron las variables influencia del compromiso afectivo de los empleados percibido por el directivo, tanto sobre su nivel de confianza como sobre la capacidad de aprendizaje organizativo (CAO) y la influencia sobre la CAO de esta predisposición del directivo a confiar en sus empleados. Se examinó si el compromiso afectivo de los empleados percibido por el directivo, la confianza del directivo y la CAO favorecen la innovación del producto, teniendo como resultado que, cuando el directivo percibe compromiso en sus empleados tiende a confiar en ellos; si el directivo percibe expectativas positivas de comportamiento en los empleados, como las que se derivan de estar afectivamente comprometido, este tenderá a adoptar posiciones de confianza frente a estos empleados, la influencia del compromiso afectivo y la confianza percibida por el directivo influyen sobre la CAO. Esto permite concluir que, si el directivo percibe compromiso en los empleados y deposita su confianza en dichos empleados, la CAO mejora y la confianza que el directivo deposita en sus empleados influye positivamente sobre el aprendizaje y en el desempeño innovador del modelo de negocio.

Otro aspecto relevante, en los resultados de este estudio, es que las dimensiones de capital intelectual tienen el siguiente orden de predicción sobre el modelo de negocio de acuerdo con los coeficientes estandarizados: CIC (Capital relacionado a la organización) es la dimensión que más influye, seguido del CIA (Capital humano) y por último el CIB (Capital estructural). Con resultados similares Ibarra Cisneros y Hernandez-Perlines (2018), efectuaron un estudio en México con el objetivo de analizar cuál de las dimensiones del capital intelectual influyen más en el desempeño manufacturero de México (esto se relaciona con el desempeño innovador de los modelos de negocio). Los resultados mostraron en sus 
coeficientes estandarizados que el mejor predictor es el capital relacional seguido del capital humano y por último el capital estructural. Los autores mencionan que estos resultados son similares a otros estudios realizados en México.

Al igual para Aramburu, Sáenz y Blanco (2015), que realizaron una investigación empírica en una población que está constituida por empresas colombianas en el área de la tecnología. Los resultados obtenidos muestran que el capital intelectual en específico la dimensión de capital estructural explica en gran medida la efectividad del proceso de generación de nuevas ideas y de la gestión de proyectos de innovación en el modelo de negocio.

Otros de los resultados obtenidos de esta investigación es que la variable que presentó la media aritmética más baja fue modelo de negocio, específicamente en la dimensión "la organización que investiga innova e involucra".

Este resultado muestra la necesidad que tiene las Pymes en México de desarrollar un modelo de negocio en donde se incluya la innovación y el involucramiento de los empleados. Esto implica prestar atención a los siguientes aspectos peor evaluados del modelo de negocio de la empresa: (a) contar con estructuras, sistemas y procedimientos que soportan la innovación, (b) otorgar incentivos económicos para los empleados que generan nuevas ideas y realizan exploración tecnológica y (c) buscar financiamiento externo para la innovación.

Con el fin de mejorar el modelo de negocio es conveniente también corregir los aspectos peor evaluados de los regresores capital intelectual y comportamiento estacional de la demanda. Para el constructo capital intelectual, se exhorta a mejorar en los siguientes aspectos: (a) evaluar de manera formal el éxito o el fracaso de la colaboración con las alianzas estratégicas, (b) contar con programas de entrenamiento para desarrollar y actualizar las competencias de los empleados constantemente y (c) hacer participar al trabajador en los programas de enriquecimiento de puesto y flexibilidad laboral. Por otro lado, en el comportamiento estacional de la demanda, se debe prestar atención a lo siguiente: (a) invertir tiempo necesario para realizar un pronóstico, (b) realizar reuniones con los clientes para efectuar trabajo colaborativo en la realización del pronóstico más preciso y (c) procurar contar con cursos de capacitación para entender la importancia de los cambios en la demanda.

Debido a la complejidad no se puedo obtener una muestra aleatoria de las Pymes que están afiliadas a CAINTRA solamente se administró el cuestionario a los directivos que estuvieron dispuestos a participar. Otra limitante con la que cuenta este estudio es la falta de investigaciones en Pymes con respecto a las variables de este estudio, ya que estas variables se han estudiado regularmente en empresas multinacionales (Börjesson, Elmquist y Hooge, 2014; Rosli y Sidek, 2013). Un ejemplo de ello es que, para las empresas grandes, la dimensión que más influye en el capital intelectual es el capital humano y en los resultados de esta investigación resulto ser el capital relacionado a la organización. 
Se recomienda para investigaciones futuras considerar el estudio en empresas ya incorporadas y operando bajo industria 4.0 para conocer si la percepción reportada presenta cambios.

\section{Referencias}

Alvarez, C. N. y Gonzales, Z. G. (2013). Determinación de las clases de capital intelectual que afectan la correcta gestión de las pymes manufactureras metalmecánicos y servicios en la ciudad de Guayaquil. Disponible en: https://dspace.ups.edu.ec/bitstream/123456789/5300/1/ UPSGT000464.pdf

Alonso, J. C. y Arcila, A. M. (2013). Empleo del comportamiento estacional para mejorar el pronóstico de un commodity: el caso del mercado internacional del azúcar. Estudios Gerenciales, 29(129), 406-415. https://doi. org/10.1016/j.estger.2013.11.006

Aramburu, N., Sáenz J. y Blanco, C. E. (2015). Structural capital, innovation capability, and company performance in technology-based Colombian firms. Cuadernos de Gestión, 15(1), 39-60. https://doi.org/10.5295/ cdg.130427na

Banco de México. (2006). Informe anual 2006. Disponible en: http://www.banxico.org.mx/documents/\%7B4EF1BFA3-67A1-F187-0DB0-243591806B5\%7D.pdf.

Bontis, N. Chua, W, \& Richardson, S. (2000). Intellectual capital and business performance in Malaysian industries. Journal of Intellectual Capital. 1 (1), 85-100 https://doi.org/10.1108/14691930010324188

Bozart, C. C., Warsing, D. P., Flynn, B. B. \& Flynn, E. J. (2009). The impact of supply chain complexity on manufacturing plant performance. Journal of Operations Management, 27, 78-93. https://doi.org/10.1016/j.jom.2008.07.003

Börjesson, S., Elmquist, M., \& Hooge, S. (2014). The challenges of innovation capability building: Learning from longitudinal studies of innovation efforts at Renault and Volvo Cars. Journal of Engineering and Technology Management, 31, 120-140. https://doi.org/10.1016/j.jengtecman.2013.11.005

Brooking, A. (1997). El Capital Intelectual. Editorial Paidós. España.

Camisón Zornoza, C., Garrigós Simón, F. J. y Palacios Marqués, D. (2007). Estrategias competitivas y desempeño empresarial: estudio comparativo de los modelos de Robinson \& Pearce y Miles \& Snow en el sector hotelero español. Investigaciones Europeas de Dirección y Economía de la Empresa, 13(3), 161-182.

Cassol, A., Reis Gonçalo, C., Santos, A. y Lima Ruas, R. (2016). A administração estratégica do capital intelectual: um modelo baseado na capacidade absortiva para potencializar inovação. Revista Ibero Americana de Estratégia, 15(1), 27-43. https://doi.org/10.5585/riae .v15i1.2161

Claver Cortés, E., Pertusa Ortega, E. y Molina Azorín, J. (2010). Estructura organizativa y resultado empresarial: análisis empírico del papel mediador de la estrategia. Cuadernos de Economía y Dirección de la Empresa, 14(1), 2-13. https://doi.org/10.1016/j.cede.2011.01.005

Cuevas Vargas, H., Aguilera Enríquez, L., González Adame, M. \& Servín, J. L. (2015). The use of ICTs and its relation with the competitiveness of Mexican SMEs. European Scientific Journal, 11(13), 294-310. https://doi. org/10.3917/resg.111.0039

De Oliveira Cabral, J. E., Fernandes Matos Coelho, A., Fernandes Coelho, F. J. \& Braga Costa, M. P. (2015). Capabilities, innovation, and overall performance in Brazilian export firms. Revista de Administração Mackenzie, 16(3), 76-108. https://doi.org/10.1590/1678-69712015-administração.v16n3p76-108

Denicolai, S., Cotta Ramusino, E., \& Sotti, F. (2015). The impact of intangibles on firm growth. Technology Analysis \& Strategic Management, 27(2), 219-236 https://doi.org/10.1080/09537325.2014.959484

Den Hertog, P., Van der Aa, W. y de Jong, M. W. (2010). Capabilities for managing service innovation: Towards a conceptual framework. Journal of Service Management, 21(4), 490-514. https://doi. org/10.1108/09564231011066123

Faust, V., Christens, B., Sparks, S. \& Hilgendorf, A. (2015) Exploring relationships among organizational capacity, collaboration, and network change. Psychosocial Intervention, 24(3), 125-131. https://doi.org/10.1016/j. 


\section{J.A. Sánchez Valdez, et al./ Contaduría y Administración 66(2) 2021, 1-20 \\ http://dx.doi.org/10.22201/fca.24488410e.2021.2295}

psi .2015 .09 .002

Garcés, E. X., López, G. M. y Pailiacho, V. M. (2017). Lienzo de modelo de negocios como herramienta de emprendimiento para personas con capacidades especiales. Un Enfoque desde la Tecnología 3C TIC, 6(2), 52-69. https://doi.org/10.17993/3ctic .2017.56.52-69

García Cruz, J. y Real Fernández, J. C. (2013). Confianza como consecuencia del compromiso percibido: implicaciones sobre el aprendizaje y la innovación. Cuadernos de Gestión, 13, 95-125. doi10.5295/cdg.110282jg

García Osorio, O., Quintero Quintero, J. y Arias Pérez, J. (2014). Capacidades de innovación, desempeño innovador y desempeño organizacional en empresas del sector servicios. Cuadernos de Administración, 27(49), 87-108. https://doi.org/10.11144/ Javeriana.cao27-49.cidi

Giraldo Marín, S. (2012). El empoderamiento como generador de compromiso organizacional en los empleados de las empresas aseguradoras de la ciudad de Manizales. (Tesis de maestría). Universidad Nacional de Colombia, Manizales, Colombia.

Hair, J., Anderson, R., Tatham, R. y Black, W. (2007). Análisis multivariante (5ª ed.). Madrid: Prentice Hall Iberia.

Ibarra-Cisneros, M. A., \& Hernandez-Perlines, F. (2018). Intellectual capital and Organization performance in the manufacturing sector of Mexico. Management Decision.

Instituto Nacional de Estadística y Geografía. (2016). Pymes, eslabón fundamental para el crecimiento en México. Disponible en: http://www.camescom.com.mx/Pymes-eslabon- fundamental-para-el-crecimiento-en-mexico/

Lavanda, G. y Rodríguez, G. (2011). Descomposición histórica de la inflación en Perú. Distinguiendo entre choques de demanda y choques de oferta. Economía, 34(67), 126-162.

Lee, H. \& Choi, B. (2003). Knowledge management enablers, processes, and organizational performance: An integrative view and empirical examination. Journal of Management Information Systems, 20(1), 179-228. https:// doi.org/10.1080/07421222.2003.11045756

Macri, D. M., Tagliaventi, M. R. \& Bertolotti, F. (2002). A grounded theory for resistance to change in a small organization. Journal of Organizational Change Management, 15(3), 292-310. https://doi. org/10.1108/09534810210429327

Manrique Henao, J. A., Robledo Velásquez, J. y Lema Tapias, Á. (2014). Índice de desempeño innovador en los subsectores industriales colombianos entre 1996 y 2005. Revista Facultad de Ciencias Económicas: Investigación y Reflexión, 22(2), 79-95. https://doi.org/10.18359/rfce.627

Martín Ríos, C. y Septiem, M. (2013). Cooperación e intercambio de conocimientos en redes inter-organizativas informales. Redes, 24(1), 193-216. https://doi.org/10.5565/rev/ redes.284

Rosli, M. M., y Sidek, S. (2013). The Impact of Innovation on the Performance of Small and Medium Manufacturing Enterprises: Evidence from Malaysia. Journal of Innovation Management in Small \& Medium Enterprises, 2013, 1-16. https://doi.org/10.5171/2013.885666

Sagástegui, J. O. (2014). El capital intelectual y su incidencia en la valorización de los activos de las Mypes de la ciudad de Trujillo (Tesis doctoral). Universidad Nacional de Trujillo, Trujillo, Perú.

Sánchez Valdez, J. A. (2018). Modelo causal entre factores del modelo innovador, la reingeniería del negocio y el desempeño empresarial validado por directivos de pymes de Monterrey, Nuevo León. (Tesis doctoral). Universidad de Montemorelos, Nuevo León, México.

Secundo, G., Dumay, J., Schiuma, G. y Passiante, G. (2016). Managing intellectual capital through a collective intelligence approach: an integrated framework for universities. Journal of Intellectual Capital, 17(2), 298-319. https://doi.org/10.1108/JIC-05-2015-0046

Spreitzer, G. M. (1995). Psychological empowerment in the workplace: Dimensions, measurement and validation. Academy of Management Journal, 38(5), 1442- 1465. https://doi.org/10.2307/256865

Villena Manzanares, F. y Souto Pérez, J. E. (2015). El impacto de los intangibles en el desempeño exportador de la Pyme manufacturera. Intangible Capital, 11, 13- 40. https://doi.org/10.3926/ic.567

Wee, J. C. y Chua, A. Y. (2016). The communication of intellectual capital: The 'whys' and 'whats'. Journal of Intellectual Capital, 17(3), 414-438 https://doi.org/10.1108/jic-01-2016-0007 


\section{Anexo}

Tabla A1

Tabla de medias aritméticas de cada ítem

\begin{tabular}{|c|c|c|}
\hline Declaraciones & Media & Desv. típ \\
\hline Conocen la misión. (RC1) & 4.11 & 1.022 \\
\hline Conocen la visión. (RC2) & 4.11 & 1.037 \\
\hline Comunican las políticas de calidad. (RC3) & 4.25 & .982 \\
\hline Establecen las metas y objetivos de su área. (RC4) & 4.44 & .799 \\
\hline Evalúan las metas. (RC5) & 4.34 & .857 \\
\hline Se recibe retroalimentación de la evaluación de las metas. (RC6) & 4.20 & .949 \\
\hline Promueve el compañerismo. (RC7) & 4.14 & .941 \\
\hline Promueve el intercambio de conocimiento. (RC8) & 4.09 & .992 \\
\hline Permite la participación activa para la solución de problemas. (RC9) & 4.25 & .856 \\
\hline Respetan los roles y normas en el grupo de trabajo. (RC10) & 4.20 & .829 \\
\hline Percibe un clima de comunicación para proponer de manera abierta soluciones. (RC11) & 4.10 & .922 \\
\hline Existe el apoyo para el crecimiento profesional y laboral. (RC12) & 3.92 & 1.038 \\
\hline Se motiva por medio de incentivos el desempeño laboral. ( $\mathrm{RC} 13)$ & 3.72 & 1.106 \\
\hline Se siente comprometido para continuar creciendo laboral y profesionalmente. (RC14) & 4.11 & 1.000 \\
\hline Está usted comprometido a alcanzar sus metas definidas por la empresa. (RC15) & 4.56 & .684 \\
\hline Se compromete para resolver problemas y desarrollar soluciones de negociación. (RC16) & 4.55 & .672 \\
\hline $\begin{array}{l}\text { Cuenta con el apoyo y entendimiento del comportamiento de la demanda (pedidos pen- } \\
\text { dientes, retrasos, inventarios, etc.). (CED1) }\end{array}$ & 4.03 & .930 \\
\hline $\begin{array}{l}\text { Imparten cursos de capacitación para entender la importancia de los cambios en la de- } \\
\text { manda. (CED2) }\end{array}$ & 3.30 & 1.172 \\
\hline $\begin{array}{l}\text { Brinda la protección adecuada a la información confidencial relacionada con la demanda } \\
\text { del cliente. (CED3) }\end{array}$ & 4.16 & 1.031 \\
\hline Invierte el tiempo necesario para realizar un pronóstico. (CED4) & 3.83 & 1.014 \\
\hline $\begin{array}{l}\text { Reúnen con los clientes para realizar trabajo colaborativo en la realización del pronós- } \\
\text { tico más preciso. (CED5) }\end{array}$ & 3.67 & 1.123 \\
\hline Colocan los pedidos y entregas de manera sencilla al cliente en tiempo y forma. (CED6) & 3.96 & .873 \\
\hline Facilita la comunicación para programar los pedidos del cliente. (CED7) & 4.14 & .893 \\
\hline Anticipan a las necesidades de una demanda alta o baja de productos o servicios. (CED8) & 4.00 & .881 \\
\hline $\begin{array}{l}\text { Cuenta con mecanismos para soportar con rapidez los cambios en la demanda de los } \\
\text { productos o servicios. (CED9) }\end{array}$ & 3.90 & .923 \\
\hline Cumplen con los acuerdos contraídos con los clientes. (CED10) & 4.26 & .742 \\
\hline
\end{tabular}


Verifica que la información que se intercambia es confiable o de calidad. (CED11)

Cuenta con tecnologías de información para el apoyo en la solución de quejas de los

Invierte en tecnologías de información para el análisis de la demanda (SAP, ORACLE,

DJ Edward). (CED13)

Cuenta con algún programa computacional para la planeación y requerimiento de ma-

teriales. (CED14)

Cuenta con indicadores en el área de trabajo que muestren el comportamiento de la

Evalúa en forma general la importancia de la necesidad de tecnología de la información.

Los empleados desarrollan ideas y conocimientos de las fuentes externas. (MN2)

El trabajador adquiere ideas y conocimientos de las fuentes externas. (MN3)

Asiste a conferencias, exposiciones y/o convenciones. (MN4)

El trabajador se involucra en la participación de investigación, desarrollo e innovaciones. (MN5)

Se capacita sobre la protección adecuada de la información o confidencialidad relacionada. (MN6)

Investiga sobre metodologías de planeación de compras estratégicas para realizarse en

Realiza investigaciones sobre la anticipación a las necesidades por tendencias y estacionalidades de la demanda. (MN9)

Contrata consultores o expertos externos para la mejora en sus procesos. (MN10)

Busca financiamiento externo para la innovación. (MN11)

Se otorgan incentivos económicos para los empleados que generan nuevas ideas y reali-

Desarrollan de forma continua proyectos de innovación (productos, procesos y/o sistemas). (MN13)

Se cuenta con estructuras, sistemas y procedimientos que soportan la innovación.

Trabajan con alianzas estratégicas en los proyectos de innovación (proveedores, clien- 
El trabajador participa en los programas de enriquecimiento de puesto y flexibilidad $\quad 3.62$

laboral. (CI3)

El empleado es capaz de desarrollar nuevas ideas y conocimiento. (CI4)

El personal hace frente a las dificultades con eficiencia. (CI5)

Fomenta la confianza y se encuentra comprometido con la empresa. (CI6)

Se cuenta con programas de entrenamiento para desarrollar y actualizar las competen-

cias de los empleados constantemente. (CI7)

Tienen procesos de reclutamiento y selección para controlar a los mejores candidatos. (CI8)

Se almacena el conocimiento y experiencias de los empleados en manuales sistemas y procesos. (CI9)

Tienen procedimientos documentados que ayudan a ejecutar acciones de rutina. (CI10)

Cuentan con la infraestructura para que los empleados accedan a información relevante.

(CI11)

Evalúa de manera formal las razones de éxito o fracaso de la colaboración con las alian-

zas estratégicas. (CI12)

Los empleados cuentan con buenas relaciones a través de redes de trabajo con clientes, proveedores, socios y amigos para el desarrollo de soluciones. (CI13)

Se aprende de las alianzas estratégicas para mejorar sus procesos. (CI14) 